\title{
CARACTERIZAÇÃo DETALHADA DE MATERIAL DE REFERÊNCIA CERTIFICADO DE CARVÃO BRASILEIRO
}

Vera L. V. Fallavena, Cristiane S. de Abreu, Taísi D. Inácio, Marçal Pires e Carla M. N. Azevedo*

Faculdade de Química, Pontifícia Universidade Católica do Rio Grande do Sul, Av. Ipiranga, 6681, 90619-900 Porto Alegre - RS, Brasil

\author{
Iolanda D. Fernandes e Lizete S. Ferret
}

Fundação de Ciência e Tecnologia, Rua Washington Luiz, 675, 90010-460 Porto Alegre - RS, Brasil

M. Rosa Martinez Tarazona

Instituto Nacional do Carvão, C/Francisco Pintado Fe, 26. 33011 Oviedo, Espanha

Recebido em 31/1/12; aceito em 7/12/12; publicado na web em 12/3/13

\begin{abstract}
DETAILED CHARACTERIZATION OF CERTIFIED REFERENCE MATERIAL OF BRAZILIAN COAL. The use and characterization of the first Certified Reference Material (CRM) of Brazilian coal for analytical quality control are presented. All results were determined within the limits of repeatability and reproducibility allowed under the standards. Notable among the characterization parameters was the mineral matter content (45.39\%) obtained by an alternative procedure showing a low relative error $(-2.1 \%)$ compared to the standard technique (low ashing temperature). The possible expanded application of this CRM application in national laboratories, beyond the certificated and reference parameters, has advantages such as lower cost and easy availability, but round robin tests must be performed.
\end{abstract}

Keywords: coal characterization; reference certified material; analytical quality.

\section{INTRODUÇÃO}

O carvão fóssil, como uma rocha sedimentar, é uma mistura heterogênea e complexa de constituintes orgânicos e inorgânicos contendo as fases sólida, líquida e gasosa intimamente misturadas. Os constituintes inorgânicos do carvão incluem os minerais, tais como cristais, grãos e agregados de vários minerais e a matéria fluida (umidade e inclusões inorgânicas) associada com constituintes inorgânicos e orgânicos sólidos. ${ }^{1} \mathrm{~A}$ caracterização físico-química do carvão apresenta vários desafios e a garantia da qualidade analítica dos dados é um fator muito importante em todas as etapas do ciclo de vida do carvão.

O carvão brasileiro apresenta características diferenciadas em relação aos carvões estrangeiros, pois contém elevado teor de cinzas e de enxofre, sendo normalmente classificado como de baixo rank. ${ }^{2,3}$ Tais características acarretam não somente problemas no seu uso, ${ }^{4}$ mas também na aplicação dos métodos/técnicas analíticas usadas em sua caracterização. ${ }^{5}$ Dentre essas dificuldades, cabe ressaltar a indisponibilidade de Materiais de Referência Certificados (MRCs) compatíveis com suas características químicas ${ }^{6}$ e geológicas, em especial seu elevado teor de matéria mineral, ${ }^{7}$ além de custos elevados. Cabe destacar que procedimentos analíticos utilizados no país foram validados com MRCs de carvões estrangeiros, com eventual aumento das incertezas associadas às determinações ${ }^{8}{ }^{89}$ Esse quadro tende a mudar através da comercialização do primeiro MRC de carvão brasileiro [CAR(MR)] disponível desde de 2009, sendo certificado para análises de cinzas, enxofre total e poder calorífico superior e apresentando valores de referência para matérias voláteis e carbono fixo. ${ }^{10}$ Entretanto, diversos outros parâmetros fazem parte das análises de rotina do carvão e não foram ainda contemplados com um MRC.

Este trabalho teve por objetivo realizar a caracterização complementar do MRC CAR(MR) visando uma aplicação mais ampla do mesmo. Essa caracterização incluiu análise elementar (CHNO),

*e-mail: cazevedo@pucrs.br diferentes formas de enxofre (pirítico, sulfático e orgânico), teor de carbonatos, composição química por espectrometria de fluorescência de Raios X (FRX) e mineralógica por difração de Raios X (DRX) e temperatura de fusão das cinzas. Foram também feitas análises térmicas, que permitiram quantificar o conteúdo de matéria mineral presente no carvão estudado.

\section{PARTE EXPERIMENTAL}

\section{Materiais de referência certificados}

Foram utilizados três diferentes lotes (09, 10 e 11) do Material de Referência Certificado de carvão CAR(MR), adquiridos junto à Rede Metrológica do RS. O MRC de carvão é oriundo da bacia carbonífera Sul Catarinense, amostrado e preparado em julho de 2009, segundo procedimento normatizado (NBR 8291 e NBR 8292). ${ }^{11,12}$ Os carvões foram certificados através do processo de comparação interlaboratorial no Programa de Ensaios de Proficiência em Análises de Carvão - $1^{\mathrm{a}}$ Rodada de 2009. ${ }^{10}$

Na Tabela 1 estão apresentados os valores de referência certificados e valores de referência para esse material conforme os certificados de análises (Rede Metrológica do RS), ${ }^{10}$ que são idênticos para os três lotes utilizados. Os teores de carbono fixo e matérias voláteis não são certificados, não sendo recomendado seu uso na determinação da exatidão dessas medidas. Esses valores de referência foram utilizados na calibração de equipamentos e no controle de qualidade analítico.

O MRC foi utilizado como recebido $(<250 \mathrm{~mm})$, sendo armazenado ao abrigo da luz e em temperatura controlada $\left(20 \pm 2{ }^{\circ} \mathrm{C}\right)$, conforme recomendações constantes no certificado. O teor de umidade de análise do padrão foi determinado ao longo do estudo, em função da necessidade de expressar os resultados em base seca.

Para verificação dos métodos de análise, outros Materiais de Referência Certificados (MRCs) e Materiais de Referência (MRs) de carvões estrangeiros foram utilizados, destacando-se padrões de carvões sul-africanos (SARM 18, SARM 19 e SARM 20), padrões 
Tabela 1. Valores de referência certificados e valores de referência para o material de referência certificado - carvão mineral CAR(MR), conforme certificado de análise

\begin{tabular}{|c|c|c|c|c|c|}
\hline Parâmetro & & Valor & $\begin{array}{l}\text { Incerteza de } \\
\text { medição }\end{array}$ & $\mathrm{k}^{\mathrm{b}}$ & Observação \\
\hline Carbono fixo & $\left(\%, b^{a}\right)$ & 40,66 & 0,73 & 2,52 & $\begin{array}{l}\text { Valor de } \\
\text { referência }\end{array}$ \\
\hline Cinzas & $(\%, \mathrm{bs})$ & 41,09 & 0,62 & 2,25 & Valor certificado \\
\hline Enxofre total & $(\%, \mathrm{bs})$ & 1,69 & 0,08 & 2,00 & Valor certificado \\
\hline Matérias voláteis & $(\%, \mathrm{bs})$ & 18,57 & 1,55 & 2,00 & $\begin{array}{l}\text { Valor de } \\
\text { referência }\end{array}$ \\
\hline $\begin{array}{l}\text { Poder calorífico } \\
\text { Superior }\end{array}$ & $(\mathrm{J} / \mathrm{g}, \mathrm{bs})$ & 19.657 & 374 & 3,31 & Valor certificado \\
\hline
\end{tabular}

abase seca, bfator de expansão.

NIST (2692b, 2693 e 2685b) e LECO (502-442, 502-443 e 502444). Nas Tabelas $1 \mathrm{~S}$ e $2 \mathrm{~S}$ (material suplementar) são apresentados os principais parâmetros certificados, de referência ou informados desses MRCs e MRs.

\section{Caracterização química e mineralógica}

Os parâmetros da análise imediata - umidade de análise, teor de cinzas, matéria volátil e carbono fixo - foram determinados seguindo procedimentos normatizados. ${ }^{13-16} \mathrm{~A}$ análise elementar do carvão envolve a determinação do percentual em massa de carbono, hidrogênio, nitrogênio, enxofre total e oxigênio (estimado por diferença). Essas análises foram realizadas em analisador elementar (Leco Corporation, modelo TruSpec CHN), segundo a norma ASTM D 5373/2008. ${ }^{17}$ As formas de enxofre (pirítico, sulfático e orgânico) foram determinadas seguindo a norma NBR 8297/1983, ${ }^{18}$ cujo método se baseia, para a determinação do enxofre sulfático existente no carvão, na dissolução seletiva desta forma de enxofre e posterior determinação gravimétrica sob forma de sulfato de bário. Para a determinação do enxofre pirítico, o método consiste na dissolução seletiva do enxofre pirítico existente no resíduo da extração do enxofre sulfático, seguido pela sua determinação a partir da análise do ferro dissolvido. O enxofre orgânico é obtido pela diferença entre o teor de enxofre total e os teores de pirítico e sulfático.

Inicialmente foram feitas análises periódicas dos parâmetros certificados/referência do CAR(MR) (Tabela 1) visando estimar as incertezas nessas determinações de rotina. As análises foram feitas em 7 replicatas e a comparação dos resultados das medições com os valores certificados foi feita seguindo procedimento da ISO/1993. ${ }^{19}$ Em todos os casos não houve diferença significativa entre o valor medido e o certificado/referência.

O teor residual de carbonatos, presente nas cinzas da amostra submetida aos diferentes tratamentos térmicos, foi determinado seguindo a norma ASTM D6316-09b/2009. ${ }^{20}$ Foram pesados entre 0,5 e $0,7 \mathrm{~g}$ (precisão de $0,1 \mathrm{mg}$ ) em cadinhos de porcelana e adicionadas 40 gotas de $\mathrm{HNO}_{3} 10 \%$. Após 90 min de reação à temperatura ambiente, a amostra foi seca em estufa à temperatura de $120^{\circ} \mathrm{C}$ por $3 \mathrm{~h}$ e determinado o teor de carbono residual no analisador elementar (Leco Truspec CHN). O teor de carbonato foi obtido por diferença entre os teores de carbono elementar determinados antes e após o ataque ácido.

Os elementos maiores constituintes das cinzas foram determinados por fluorescência de raios X (ASTM D4326-94/1996)21 utilizando equipamento Rigaku RIX 2000 com fonte de radiação de ródio. A determinação da composição mineral de amostras de carvão foi realizada em difratômetro de raios X (Rigaku Dengi D-Max 2000), com radiação $\mathrm{Cu} \mathrm{Kq}$, gerada a $30 \mathrm{kV}$ e $15 \mathrm{~mA}$. As fases minerais foram identificadas com o auxílio do aplicativo Jade Plus 5.

\section{Análise térmica e determinação do teor de matéria mineral}

Foram realizadas análises térmicas utilizando um analisador térmico simultâneo TG-DTA (TA Instruments, SDT Q600). As amostras com massa entre 10-15 mg foram colocadas em um cadinho de platina e aquecidas a uma taxa de aquecimento de $20{ }^{\circ} \mathrm{C} \min ^{-1}$ de 25 até $1.000{ }^{\circ} \mathrm{C}$, sob fluxo de $\mathrm{N}_{2}$ a uma vazão de $100 \mathrm{~mL} \mathrm{~min}^{-1}$. Depois de atingida a temperatura de $1.000{ }^{\circ} \mathrm{C}$, a atmosfera foi trocada para ar e mantida uma isoterma de $30 \mathrm{~min}$. As curvas de DTA e DTG foram obtidas através de utilização do aplicativo TA Universal Analysis.

Foram feitos testes de fusibilidade das cinzas do MRC CAR(MR) obtidas $\left(800 \pm 20^{\circ} \mathrm{C}, 3-4 \mathrm{~h}\right.$ ) para verificar o comportamento dos componentes inorgânicos do carvão durante o aquecimento. Foram determinadas as temperaturas: inicial de deformação, esfera e/ou de amolecimento, de hemiesfera e fluidez, em função das deformações específicas decorrentes da transição de fase nos corpos de prova. Esses testes foram realizados em microscópio de aquecimento 1.750 ${ }^{\circ} \mathrm{C}$ (marca Hesse Instruments, modelo HT19 (1750/80)), com placa de captura de imagens e software para análise de imagens) de acordo com a norma DIN 51730, ${ }^{22}$ com um corpo de prova (cilíndrico $3 \mathrm{x}$ $3 \mathrm{~mm}$, atmosfera oxidante $-\mathrm{O}_{2}$ ). $\mathrm{O}$ ensaio foi realizado com programação até a capacidade máxima da temperatura do forno que é 1750 ${ }^{\circ} \mathrm{C}$ e temperatura máxima da amostra que é $1.650^{\circ} \mathrm{C}$. Os resultados expressos na Tabela 2 representam a média aritmética de duas vias, enquanto a Figura 1S (material suplementar) apresenta imagens geradas do ensaio de uma das vias.

Tabela 2. Resultados das análises imediata e elementar, formas de enxofre do MRC CAR(MR) e composição química de suas cinzas (HTA $775^{\circ} \mathrm{C}$ )

\begin{tabular}{|c|c|c|c|}
\hline \multicolumn{2}{|l|}{ Carvão } & \multicolumn{2}{|c|}{ Cinza do carvão } \\
\hline \multicolumn{2}{|c|}{ Análise imediata $\left(\%, b^{a}\right)$} & \multicolumn{2}{|c|}{ Composição química (\%) } \\
\hline Umidade $(\%)^{\mathrm{b}}$ & 1,03 & $\mathrm{SiO}_{2}$ & 57,80 \\
\hline Cinzas & 41,19 & $\mathrm{Al}_{2} \mathrm{O}_{3}$ & 29,06 \\
\hline Matéria volátil & 19,17 & $\mathrm{TiO}_{2}$ & 1,40 \\
\hline Carbono fixo $^{c}$ & 39,64 & $\mathrm{Fe}_{2} \mathrm{O}_{3}$ & 4,80 \\
\hline \multicolumn{2}{|c|}{ Análise elementar $(\%$, bs $)$} & $\mathrm{MgO}$ & 0,47 \\
\hline Carbono total & 49,47 & $\mathrm{CaO}$ & 1,44 \\
\hline Hidrogênio & 3,12 & $\mathrm{Na}_{2} \mathrm{O}$ & 0,16 \\
\hline Nitrogênio & 0,85 & $\mathrm{~K}_{2} \mathrm{O}$ & 2,72 \\
\hline$\left(\right.$ Oxigênio + Halogênios) ${ }^{c}$ & 5,37 & $\mathrm{P}_{2} \mathrm{O}_{5}$ & $<0,03$ \\
\hline Enxofre total & 1,62 & $\mathrm{SO}_{3}$ & 1,06 \\
\hline \multicolumn{2}{|c|}{ Formas de enxofre $(\%, \mathrm{bs})$} & $\mathrm{LOI}^{\mathrm{d}}$ & 1,06 \\
\hline Enxofre pirítico & 0,82 & \multicolumn{2}{|c|}{ Fusibilidade das cinzas $\left({ }^{\circ} \mathrm{C}\right)$} \\
\hline Enxofre sulfático & 0,31 & Deformação & 1.539 \\
\hline Enxofre orgânico ${ }^{c}$ & 0,49 & Esfera & 1.595 \\
\hline \multicolumn{2}{|c|}{ Poder calorífico superior } & Hemiesfera & 1.616 \\
\hline$(\mathrm{J} / \mathrm{g}, \mathrm{bs})$ & 19.573 & Fluidez & $>1.650$ \\
\hline
\end{tabular}

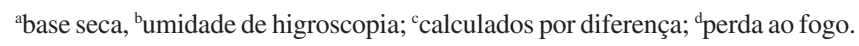
As análises foram feitas usando o CAR(MR) lote 09/09.

A estimativa do teor de matéria mineral no MRC CAR(MR) foi obtida a partir da análise de cinzas em média e baixa temperaturas e pela aplicação da fórmula de Parr. ${ }^{5}$ Três diferentes temperaturas $\left(300,370\right.$ e $\left.400{ }^{\circ} \mathrm{C}\right)$ foram estudadas na combustão da amostra $(1,5$ 
g) utilizando forno mufla (modelo EDG-3000-1P). Os períodos de queima variaram em função das temperaturas num intervalo de 20 a 382 h. Para comprovar a queima total da matéria orgânica foram realizadas determinações dos teores de carbono orgânico nas amostras tratadas termicamente.

A determinação de cinzas à baixa temperatura (LTA, Low ashing temperature), utilizando um plasma de oxigênio eletronicamente excitado $\left(<200^{\circ} \mathrm{C}\right)$, foi realizada no INCAR (Espanha) em um equipamento Emitech K 1050X. Os resíduos de LTA foram usados na caracterização dos minerais por difração de raios X.

A fórmula de Parr (Equação 1), que requer apenas valores dos teores percentuais de cinzas à alta temperatura $(\mathrm{Cz})$ e enxofre total (S), determinados na análise de rotina, ${ }^{2,5}$ foi usada numa estimativa preliminar do teor de matéria mineral (MM) do padrão em estudo.

$$
\% \mathrm{MM}=1,08 . \mathrm{Cz}+0,55 . \mathrm{S}
$$

\section{RESULTADOS}

\section{Caracterização química e mineralógica do MRC CAR(MR)}

Na Tabela 2 são apresentados diversos dados de caracterização do MRC CAR(MR) obtidos. Os resultados da análise imediata foram comparados com os valores certificados e valores de referência (Tabela 1). No que concerne aos teores de cinzas, observou-se o menor erro absoluto $(-0,1 \%)$ correspondendo a um erro percentual de $0,24 \%$, indicando uma boa exatidão. O erro absoluto verificado na determinação das matérias voláteis foi maior $(-0,6 \%)$ devido à maior complexidade dessa análise, correspondendo a um erro percentual de $3,23 \%$. Já o erro percentual na estimativa do carbono fixo foi de $-2,51 \%$, correspondendo a $1,02 \%$ de diferença absoluta, que retrata o somatório das incertezas na determinação dos demais parâmetros, utilizados no seu cálculo. Uma vez que os valores do carbono fixo e as matérias voláteis são de referência, a comparação com os dados experimentais deve ser utilizada para controle analítico. Para todos os parâmetros não há diferença significativa entre o valor medido e o certificado/referências.

O teor de enxofre total obtido $(1,62 \%)$ é similar ao valor certificado $(1,69 \%)$ com diferença de $0,07 \%$, menor que o tolerado $(0,1 \%)$ para carvões com teores de enxofre até2 \% (ASTM D3177/02/2007). ${ }^{23}$ $\mathrm{Na}$ Tabela 2 observa-se também que o poder calorífico superior determinado $(19.573 \mathrm{~J} / \mathrm{g}$ ) foi próximo ao valor certificado (19.657 $\mathrm{J} / \mathrm{g}$ ) com diferença de $84 \mathrm{~J} / \mathrm{g}$ e dentro do limite de reprodutibilidade estabelecido $\left(<250 \mathrm{~J} / \mathrm{g}\right.$ ) pela norma (ASTM D5865/2010). ${ }^{24}$

A análise elementar indicou teor de carbono $(49,47 \%)$ compatível com o tipo de carvão estudado e com o teor de cinzas da amostra $(41,19 \%)$. Os teores de hidrogênio $(3,12 \%)$ e nitrogênio $(0,85 \%)$ estão dentro da faixa esperada para o grau de carbonificação dessa amostra. ${ }^{2}$ Cabe ainda destacar que as incertezas associadas a essas determinações estão dentro da faixa tolerada para as normas utilizadas. ${ }^{17}$

Os teores das formas de enxofre também são mostrados na Tabela 2. A amostra CAR(MR) apresentou maiores concentrações de enxofre pirítico $(0,82 \%)$, seguido do enxofre orgânico $(0,49 \%)$ e teores mais baixos, porém significativos, de enxofre na forma sulfática $(0,31 \%)$. Os carvões com elevados teores de enxofre total usualmente contêm esse elemento em combinação inorgânica, onde a forma de sulfeto de ferro (pirita ou marcassita) é predominante, incluindo também outros sulfetos e sulfatos metálicos. ${ }^{25}$ Nos carvões brasileiros observa-se que o enxofre se encontra predominantemente sob a forma de pirita. Entretanto, a comparação dos valores relativos entre as três formas de enxofre observada no padrão $\mathrm{CAR}(\mathrm{MR})$ indica proporção de enxofre orgânico (30\%) muito maior que a observada em outros carvões brasileiros. ${ }^{7}$ De acordo com Calkins, ${ }^{25}$ o enxofre orgânico é o mais difícil de ser removido nos processos de beneficiamento do carvão (baseados em densidade ou lavagem), pois este é parte da própria matriz carbonosa. $\mathrm{O}$ enxofre orgânico também é a forma mais complexa e difícil de ser identificada e caracterizada. ${ }^{5}$

A cinza obtida à alta temperatura (HTA, $775^{\circ} \mathrm{C}$ ) da amostra CAR(MR) teve sua composição química determinada por fluorescência de Raios X e as características de fusibilidade das cinzas determinadas em microscópio de aquecimento. Os resultados obtidos estão apresentados na Tabela 2 e demonstram que os óxidos de $\mathrm{Si}$ (57,8\%), $\mathrm{Al}(29,06 \%)$ e Fe (4,8\%) são predominantes nessa amostra. Cabe salientar que o carvão CAR(MR) não é uma amostra típica em termos de concentração de ferro, o qual se apresenta em concentrações menos elevadas do que as reportadas para carvões brasileiros. Fallavena ${ }^{7}$ verificou concentrações 3 a 5 vezes menores de Fe para a amostra CAR(MR) comparada a outros 5 carvões ROM de SC e RS. Outros óxidos importantes encontrados foram os de K $(2,72 \%)$ e de Ti $(1,4 \%)$, além do enxofre residual $\left(\mathrm{SO}_{3} 1,06 \%\right)$. Os altos conteúdos de silício e alumínio estão relacionados às fases minerais majoritárias (aluminossilicatos), identificadas por DRX e que serão discutidas a seguir.

Os teores mais baixos de enxofre e de ferro observados podem estar relacionados à segregação dos minerais contendo esses elementos durante um provável pré-tratamento da amostra através de jigagem - separação por meio denso cujo princípio é a diferença de densidade entre os componentes do carvão. Outra indicação do possível beneficiamento desse carvão é seu poder calorífico relativamente elevado $\sim 19.600 \mathrm{~J} / \mathrm{g}$ (equivalente a um carvão energético CE4700) quando comparado a carvões brutos brasileiros. ${ }^{26}$ Cabe destacar que não é incomum o uso de carvões beneficiados como MRC, como é o caso dos padrões NIST 2693 e 2692 b, utilizados nesse estudo.

\section{Análise térmica do MRC CAR(MR)}

Na Tabela 2 são apresentadas as características de fusibilidade das cinzas. Esse parâmetro tem grande importância tecnológica, pois a formação de escória e a consequente diminuição da eficiência dos processos de combustão podem ser previstas por essa análise. Os resultados obtidos para o carvão CAR(MR) indicaram todas as temperaturas de fusibilidade superiores às relatadas na literatura para carvões brasileiros, ${ }^{27,28}$ indicando uma cinza com propriedades refratárias.

A Figura 1 mostra as curvas termogravimétricas (TG) e termogravimétrica derivada (DTG). A curva TG mostra uma diminuição de massa relativa à perda de umidade $(0,8 \%)$ seguida de uma perda

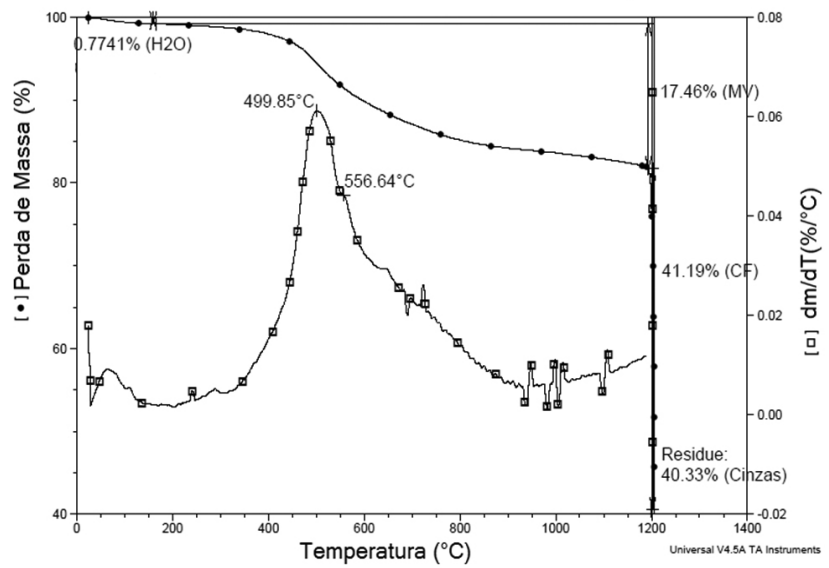

Figura 1. Curvas de análise térmica (TG, DTG) da amostra de carvão CAR(MR) em atmosfera de nitrogênio com fluxo de $20^{\circ} \mathrm{C} \mathrm{min}^{-1}$ até temperatura de $1000{ }^{\circ} \mathrm{C}$, seguida de troca para ar com isoterma de $30 \mathrm{~min}$ 
por liberação de voláteis $(17,4 \%)$ em atmosfera inerte $\left(\mathrm{N}_{2}\right)$. Após a troca para atmosfera oxidante (ar) a amostra sofreu combustão, indicada pela rápida perda de massa relativa ao carbono fixo $(41,19 \%)$ queimado. A massa restante $(40,33 \%)$ corresponde à quantidade residual de cinzas. Como esperado, esses valores estão na faixa dos resultados obtidos na análise imediata (Tabela 2).

Na curva DTG, o pico maior (em torno de $499{ }^{\circ} \mathrm{C}$ ) é provavelmente constituído por vários processos de pirólise. Segundo Zoller e colaboradores ${ }^{29}$ esse pico pode corresponder à clivagem de ligações no interior da rede macromolecular de carvão e de dessorção térmica dos compostos de estruturas termicamente menos estáveis. O ombro observado próximo a $557^{\circ} \mathrm{C}$ é identificado como uma decomposição térmica da pirita ${ }^{30,31}$ Segundo Calkins e colaboradores, ${ }^{25}$ este pico é ausente em carvões que não contêm pirita ou que apresentam esse mineral em concentrações baixas. Este resultado está de acordo com os dados obtidos na determinação das formas de enxofre, que indicaram teor de $0,81 \%$ de enxofre pirítico.

\section{Perfis de queima do MRC CAR(MR) e caracterização das cinzas obtidas}

A Figura 2 apresenta os perfis de variação percentual da massa do MRC CAR(MR) em três diferentes temperaturas de combustão em tratamento térmico convencional (forno mufla). Observa-se uma perda de massa muito rápida para as temperaturas de 370 e 400 ${ }^{\circ} \mathrm{C}$, nas primeiras horas de aquecimento, não ocorrendo variações significativas $(\mathrm{Dm}<0,5 \%)$ após $20 \mathrm{~h}$ de teste. Por outro lado, para a temperatura de $300{ }^{\circ} \mathrm{C}$ a perda de massa é muito mais lenta, sendo necessárias $382 \mathrm{~h}$ para término do teste. Observou-se também maior variabilidade entre as replicatas na menor temperatura estudada.

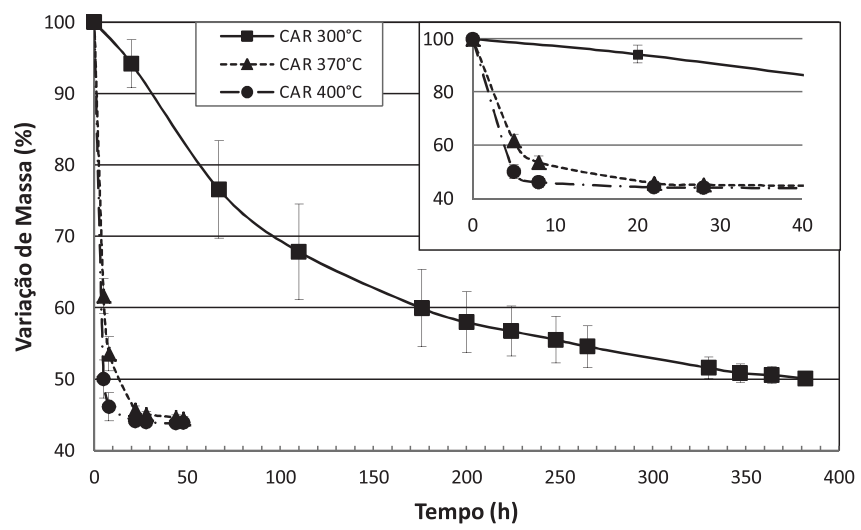

Figura 2. Perfil da variação de massa das amostras de carvão submetidas a tratamento térmico nas temperaturas estudadas (300, 370 e $\left.400{ }^{\circ} \mathrm{C}\right)$, em função do tempo

Apesar do maior tempo de aquecimento a $300{ }^{\circ} \mathrm{C}$, o valor de cinzas residual $(50,09 \%)$ é maior que os obtidos nas temperaturas mais elevadas de $370{ }^{\circ} \mathrm{C}(44,48 \%)$ e $400{ }^{\circ} \mathrm{C}(43,91 \%)$. Esse resultado se deve a dois aspectos: de um lado, à possibilidade de combustão incompleta da matéria orgânica, em particular nas temperaturas médias de combustão utilizadas e, de outro, às transformações térmicas de diferentes componentes minerais do carvão. Ambos os fenômenos podem acarretar em estimativas erradas da matéria mineral do carvão, tanto pela superestimação desconsiderando o carbono orgânico incombusto, quanto pela subestimação das fases minerais perdidas no aquecimento (por ex., pirita).

Os difratogramas de Raios X das amostras tratadas termicamente (Figura 2S, material suplementar) confirmam o desaparecimento e/ ou perda de intensidade significativa nos picos atribuídos à calcita e pirita, quando comparados às amostras brutas. Tal comportamento não era esperado para o tratamento a $370{ }^{\circ} \mathrm{C}$, usualmente aceito como procedimento de referência. ${ }^{30,32} \mathrm{~A}$ amostra bruta apresenta, como fases majoritárias, quartzo $\left(\mathrm{SiO}_{2}\right)$, caolinita $\left(\mathrm{Al}_{4}(\mathrm{OH})_{8}\left(\mathrm{Si}_{4} \mathrm{O}_{10}\right)\right.$, muscovita $\left(\mathrm{KAl}_{2} \mathrm{Si}_{3} \mathrm{AlO}_{10}(\mathrm{OH})_{2}\right.$ e pirita $\left(\mathrm{FeS}_{2}\right)$. Já nas amostras de cinzas são observados também a calcita $\left(\mathrm{CaCO}_{3}\right)$, sulfato de cálcio hemi-hidratado $\left(\mathrm{CaSO}_{4} \cdot\left(\mathrm{H}_{2} \mathrm{O}\right)_{0,5}\right.$ e ortoclásio $\left(\mathrm{KAlSi}_{3} \mathrm{O}_{8}\right)$. No que concerne à calcita, a não visualização no difratograma da amostra bruta pode estar relacionada com a influência da matéria orgânica amorfa, relatada em outros estudos. ${ }^{28}$ Entretanto, o aumento de intensidade do pico da calcita com o incremento de temperatura nos tratamentos térmicos sugere, também, uma possível formação desse mineral pela reação entre os óxidos de cálcio e o $\mathrm{CO}_{2}$ oriundo do processo de queima.

De modo geral, os resultados obtidos estão concordantes com dados de literatura, que citam argilas, sulfetos, carbonato e quartzo como os minerais mais comuns nos carvões. ${ }^{2,3,33}$ A hematita $\left(\mathrm{Fe}_{2} \mathrm{O}_{3}\right)$ é identificada nas cinzas a $400{ }^{\circ} \mathrm{C}$ representando uma provável oxidação da pirita, não observada nessa amostra de cinzas. Apesar das dificuldades na avaliação das transformações minerais, pode-se sugerir que a amostras tratada a $300{ }^{\circ} \mathrm{C}$ mantém maior similaridade com a amostra bruta, não ocorrendo descaracterização significativa dos minerais originalmente presentes.

Os teores de carbono residual e de carbonatos determinados nas cinzas obtidas nas diferentes temperaturas de calcinação são apresentados na Tabela 3. O percentual de carbono residual acompanha a tendência dos teores de cinza, atingindo valor significativo no tratamento térmico à mais baixa temperatura $(6,64 \%)$. Valores menores foram observados a 370 e $400{ }^{\circ} \mathrm{C}$, indicando uma maior oxidação da matéria orgânica. Infelizmente a maior eficiência de combustão é acompanhada de transformação mais intensa nos minerais originais do carvão, como verificado nas análises de DRX.

Tabela 3. Teores de cinzas, de carbono residual e carbonatos da amostra CAR(MR) nos diferentes tratamentos térmicos $\left(300,370\right.$ e $\left.400{ }^{\circ} \mathrm{C}\right)$

\begin{tabular}{cccccc}
\hline Teste & $\begin{array}{c}\mathrm{T} \\
\left({ }^{\circ} \mathrm{C}\right)\end{array}$ & $\begin{array}{c}\text { HTA } \\
\left(\% \mathrm{bs}^{\mathrm{a}}\right)\end{array}$ & \multicolumn{2}{c}{ Carbono residual $\left(\%, \mathrm{bs}^{\mathrm{a}}\right)$} & \begin{tabular}{c} 
Carbonatos \\
\cline { 5 - 6 }
\end{tabular} \\
\hline MTA & 300 & 50,09 & 6,64 & 4,70 & 7,11 \\
& 370 & 44,48 & 1,69 & 1,37 & 1,17 \\
& 400 & 43,91 & 0,87 & 0,62 & 0,92 \\
\hline
\end{tabular}

abase seca; as análises foram feitas usando o CAR(MR) lote 09/09.

Esse comportamento pode ser melhor quantificado através da determinação do carbono inorgânico nessas cinzas, expresso na forma de carbonatos $\left(\% \mathrm{CO}_{2}\right)$. Verifica-se que parte significativa $(30 \%)$ do carbono residual das cinzas calcinada a $300{ }^{\circ} \mathrm{C}$ corresponde a carbonatos $(7,11 \%)$. Nas demais temperaturas, o teor de carbonatos decresce atingindo $0,92 \%$, o que confirma a provável descaracterização de algumas fases minerais sob tratamento térmico mais intenso. Observa-se que a matéria orgânica incombusta $\left(\mathrm{C}_{\text {total }}-\mathrm{C}_{\text {inorgânico }}\right)$ é significativa, correspondendo a $4,7 \%$ das cinzas obtidas no teste a $300{ }^{\circ} \mathrm{C}$. Esses resultados indicam que a estimativa do teor $\mathrm{MM}$ do MRC CAR(MR) é suscetível à maior incerteza se o teor de carbono orgânico não for determinado.

\section{Análise de cinzas à baixa temperatura (LTA)}

A LTA é indicada por vários autores ${ }^{1,2,32,34,35}$ como sendo capaz de isolar a fração inorgânica, com pequenas alterações de sua forma original. Os resultados da aplicação da técnica à amostra CAR(MR) 
indicaram teor de cinzas de 46,38\%. Cabe destacar que não foram observadas diferenças significativas nas formas minerais identificadas (DRX) para a amostra bruta e a amostra tratada LTA (Figura $2 \mathrm{~S}$, material suplementar). Isso ocorre porque o carvão tem um alto conteúdo de matéria mineral. Para carvão com menor teor de cinzas, a identificação direta dos minerais por DRX é dificultada pela interferência da matéria orgânica presente.

Entretanto, a técnica LTA demanda um longo tempo para a queima total da matéria orgânica, havendo possibilidades de pequenas alterações de algumas fases minerais. ${ }^{33}$ Cabe destacar que essas transformações de minerais podem também ocorrer no tratamento térmico à média temperatura (MTA $300{ }^{\circ} \mathrm{C}$ ). Outra desvantagem da técnica de LTA é a pequena quantidade de amostra analisada, necessitando um número grande de replicatas para a obtenção de uma massa de cinzas que possa ser usada em testes de caracterização.

\section{Estimativa do teor de matéria mineral}

A Tabela 4 apresenta a comparação dos teores de MM estimados a partir da fórmula de Parr, com os teores de cinzas à alta (HTA), baixa (LTA) e média (MTA $300^{\circ} \mathrm{C}$ ) temperaturas, com e sem correção do carbono residual. Como esperado, o teor de cinzas à alta temperatura $(40,88 \%)$ não é um bom parâmetro para a estimativa do teor de MM apresentando erro de $-11,9 \%$, considerando o valor LTA como de referência. Esses valores confirmam as transformações significativas dos minerais sofridas na temperatura do teste.

Tabela 4. Teores de cinzas à baixa (LTA), média (MTA) e alta (HTA) temperaturas e o teor de matéria mineral (MM) obtido pela correlação de Parı

\begin{tabular}{ccccc}
\hline Método & Temperatura & Condição & Média & Erro $\left.^{\mathrm{a}} \%\right)$ \\
\hline HTA & $775{ }^{\circ} \mathrm{C}$ & & 40,88 & $-11,9$ \\
MM & Parr & & 45,00 & $-3,0$ \\
MTA & $300{ }^{\circ} \mathrm{C}$ & Sem correção & 50,09 & 8,0 \\
& & Correção/ $C_{\text {total }}$ & 43,45 & $-6,3$ \\
& & Correção/ $\mathrm{C}_{\text {orgânico }}$ & 45,39 & $-2,1$ \\
LTA & \multirow{2}{*}{$150{ }^{\circ} \mathrm{C}$} & & 46,38 & - \\
\hline
\end{tabular}

${ }^{a}$ Erro percentual relativo calculado considerando o valor da LTA como referência. As análises foram feitas usando o CAR(MR) lote 09/09.

A comparação do valor obtido pelo MTA a $300{ }^{\circ} \mathrm{C}$ sem correção com a LTA apresenta diferença positiva significativa $(8,0 \%)$. Por outro lado, a correção desse valor pelo desconto do teor de carbono total, medido nas cinzas residuais, subestima o teor de MM (erro de $-6,3 \%)$. O menor erro $(2,1 \%)$ nessa estimativa foi obtido através da correção do valor MTA com o teor da matéria orgânica incombusta $\left(\mathrm{C}_{\text {total }}-\mathrm{C}_{\text {inorgânico }}\right.$, Tabela 3$)$. Como tanto o teor de carbono residual quanto o teor de carbonatos no resíduo são elevados nessa amostra, a simples subtração do carbono incombusto do resíduo da calcinação não é adequada. Dessa forma, a avaliação correta do nível de matéria mineral nessa amostra de carvão deve ser feita após a determinação dos teores de carbono total e carbono inorgânico.

A estimativa do teor de matéria mineral pela fórmula de Parr $(45,00 \%)$ foi inferior ao teor de cinzas à baixa temperatura LTA $(46,38 \%)$, apresentando erro relativo de $-3,0 \%$. O LTA é considerado como o método mais adequado na estimativa do teor de MM. Entretanto, são relatadas algumas dificuldades em sua aplicação para carvões de baixo rank, como o desse estudo. Levando em consideração as limitações dos métodos utilizados, é possível propor a estimativa do teor de $\mathrm{MM}$, através de tratamento térmico à média temperatura $\left(300{ }^{\circ} \mathrm{C}\right.$, mínimo $\left.382 \mathrm{~h}\right)$ com correção da matéria orgânica incombusta.

\section{CONCLUSÃO}

Todos os parâmetros certificados, bem como os valores de referências, do MRC CAR(MR) foram determinados dentro dos limites de repetitividade e reprodutibilidade, admitidos nas respectivas normas. O uso do MRC CAR(MR), na verificação rotineira no controle de qualidade analítica de ensaios, apresenta vantagens como menor custo, disponibilidade e resultados analíticos dentro das faixas de concentração características do carvão fóssil brasileiro.

Dentre os diversos parâmetros de caracterização cabe destacar o conteúdo de matéria mineral $(45,39 \%)$ estimado por tratamento térmico à baixa temperatura $\left(300^{\circ} \mathrm{C}\right)$. Esse procedimento alternativo apresentou erro relativo baixo $(-2,1 \%)$, quando comparado à técnica padrão LTA $(46,38 \%)$, desde que sejam feitas as correções referentes à presença do carbono orgânico residual, que foi significativo $(4,7 \%)$ para o CAR(MR).

Os resultados da caracterização detalhada do CAR(MR) contribuem para um melhor conhecimento desse MRC e de sua mais ampla aplicação na caracterização de carvões brasileiros. Os diversos valores informados podem também servir como guia para a escolha de novos parâmetros de certificação por meio de testes interlaboratoriais independentes.

\section{MATERIAL SUPLEMENTAR}

Estão disponíveis em http://quimicanova.sbq.org.br, na forma de arquivo PDF, com acesso livre, o comportamento da cinza de carvão em teste de fusibilidade e os difratogramas obtidos para a amostra CAR(MR) bruta e após tratamento em diferentes temperaturas. Também estão disponíveis os valores certificados, de referência e informados dos Materiais de Referência Certificados e Materiais de Referência de carvões estrangeiros utilizados.

\section{AGRADECIMENTOS}

À Rede Carvão CNPq e à FAPERGS pelo financiamento do projeto. Ao CEPAC/PUCRS pela realização de análises elementares.

\section{LISTA DE ABREVIATURAS}

ABNT - Associação Brasileira de Normas Técnicas

ASTM - American Society for Testing and Materials

bs - base seca

CAR(MR) - Material de Referência Certificado de carvão brasileiro $\mathrm{CF}$ - Carbono fixo

$\mathrm{C}_{\text {inorgânico }}$ - Carbono inorgânico

$\mathrm{C}_{\text {orgânico }}-$ Carbono orgânico

$\mathrm{C}_{\text {total }}$ - Carbono total

$\mathrm{Cz}$ - Teor de cinzas

DRX - Difração de Raios X

DTA - Análise Térmica Diferencial, Differential Thermal Analysis

DTG - Termogravimetria Derivada

FRX - Espectrometria de Fluorescência de Raios X

HTA - Teor de cinzas à alta temperatura, High Temperature Ashing

ISO - International Organization for Standardization

k - Fator de expansão

LOI - Perda por ignição, Loss on Ignition

LTA - Teor de cinzas à baixa temperatura, Low Temperature Ashing

MM - Matéria Mineral

MR - Material de Referência

MRC - Material de Referência Certificado

MTA - Teor de cinzas à média temperatura

MV - Matérias voláteis 
NIST - National Institute of Standards and Technology

PCS - Poder Calorífico Superior

ROM - Carvão bruto, Run of Mine

SARM - South African Reference Materials

TG - Termogravimetria

VC - Valor Certificado

VI - Valor Indicado

VR - Valor de Referência

Dm - Variação de massa das amostras de carvão e cinzas nos testes de análise térmica

\section{REFERÊNCIAS}

1. Vassilev, S. V.; Tascón, J. M. D.; Energy Fuels 2003, 17, 271.

2. Nahuys, J.; Alpern, B.; Martinez, L.; Comunicações dos Serviços Geológicos de Portugal 1984, 70, 299.

3. Kalkreuth, W.; Holz, M.; Kern, M.; Machado, G.; Mexias, A.; Silva, M. B.; Willett, J.; Finkelman, R.; Burger, H.; Int. J. Coal Geol. 2006, 68, 79.

4. Flues, M.; Sato, I. M.; Cotrim, M. B.; Figueiredo Filho, P. M.; Camargo, I. M. C.; Quim. Nova 2008, 31, 25.

5. Speight, J. G.; Handbook of Coal Analysis, John Wiley \& Sons: New Jersey, 2005.

6. Sánchez, J. C. D.; Fernandes, I. D.; Mergel, N. M. D. Em Meio Ambiente e Carvão - Impactos da exploração e utilização; Governo do Estado do Rio Grande do Sul, Secretaria do Meio Ambiente; Fundação Estadual de Proteção Ambiental: Porto Alegre, 2002, cap. 9.

7. Fallavena,V. L. V.; Tese de Doutorado, Pontifícia Universidade Católica do Rio Grande do Sul, Brasil, 2011.

8. Pires, M.; Querol, X.; Int. J. Coal Geol. 2004, 60, 57.

9. Silva, L. F. O.; Izquierdo, M.; Querol, X.; Finkelman, R. B.; Oliveira, M. L. S.; Wollenschlager, M.; Towler, M.; Pérez-López, R.; Macias, F.; Environ. Monit. Assess. 2011, 175, 109.

10. Rede Metrológica RS; Certificado de Análises do Material de Referência Certificado - Carvão mineral, $C A R(M R)$, Associação Rede de Metrologia e Ensaios do Rio Grande do Sul: Porto Alegre, 2011.

11. NBR 8291; Amostragem de carvão mineral bruto elou beneficiado, Associação Brasileira de Normas Técnicas (ABNT): Rio de Janeiro, 1983.

12. NBR 8292; Preparação de amostra de carvão mineral para análise e ensaios, Associação Brasileira de Normas Técnicas (ABNT): Rio de Janeiro, 1983

13. NBR 8293; Carvão Mineral Determinação de Umidade, Associação Brasileira de Normas Técnicas (ABNT): Rio de Janeiro, 1983.

14. NBR 8289; Carvão Mineral Determinação do Teor de Cinzas, Associação Brasileira de Normas Técnicas (ABNT): Rio de Janeiro, 1983.

15. NBR 8290; Carvão Mineral Determinação do Teor de Matérias Voláteis, Associação Brasileira de Normas Técnicas (ABNT): Rio de Janeiro, 1983.
16. ASTM D 3172/0a; Standard Practice for Proximate Analysis of Coal and Coke, American Society for Testing and Materials (ASTM): Pennsylvania, 2007.

17. ASTM D 5373/2008; Standard Test Methods for Instrumental Determination of Carbon, Hydrogen, and Nitrogen in Laboratory Samples of Coal and Coke, American Society for Testing and Materials (ASTM): Pennsylvania, 2008.

18. NBR 8297; Carvão mineral - Determinação dos teores das diferentes formas de enxofre, Associação Brasileira de Normas Técnicas (ABNT): Rio de Janeiro, 1983.

19. ISO; Guide to the expression of uncertainty in measurement, Organização Internacional de Normalização (ISO): Genebra, 1993.

20. ASTM D6316-09b; Determination of combustible and carbonate carbon in solid residues from coal and coke, American Society for Testing and Materials (ASTM): Pennsylvania, 2009.

21. ASTM D 4326/11; Test Method for Major and Minor Elements in Coal and Coke Ash by X-Ray Fluorescence, American Society for Testing and Materials (ASTM): Pennsylvania, 1996.

22. DIN 51730/2007/09; Determination of fusibility of fuel ash, German Institute for Standardization (DIN): Berlin, 1998.

23. ASTM D3177-02; Standard test Methods for Total Sulfur in the Analysis Sample of Coal and Coke, American Society for Testing and Materials (ASTM): Pennsylvania, 2002.

24. ASTM D5865-10; Standard Test Method for Gross Calorific Value of Coal and Coke, American Society for Testing and Materials (ASTM): Pennsylvania, 2010.

25. Calkins, W. H.; Fuel 1994, 73, 475.

26. Müller, A. A.; Santos, H. M; Schmitt, J. C. C.; Maciel, L. A. C; Bertol, M. A.; César, S. B.; Perfil Analítico do Carvão, Departamento Nacional da Produção Mineral: Porto Alegre, 1987.

27. Redivo, R. V.; Dissertação de Mestrado, Universidade de São Paulo, Brasil, 2002.

28. Silva, N. I. W.; Calarge, L. M.; Chies, F.; Mallmann, J. E.; Zwonok, O.; Cerâmica 1999, 45, 256.

29. Zoller, D. L.; Johnston, M. V.; Tomic, J.; Wang, X.; Calkins, W. H.; Energy Fuels 1999, 13, 1097.

30. Mukherjee, S.; Srivastava, S. K.; Energy Fuels 2006, 20, 1089.

31. da Silva Filho, C. G.; Milioli, F. E.; Quim. Nova 2008, 31, 98.

32. Pinetown, K. L.; Ward, C. R.; Westhuizen, W. V.; Int. J. Coal Geol. 2007 $70,166$.

33. Ward, C. R.; López, I. C.; Int. J. Coal Geol. 2008, 73, 3.

34. Ward, C. R.; Int. J. Coal Geol. 2002, 50, 135.

35. Pusz, S.; Krzton, A.; Komraus, J. L.; Martínez-Tarazona, M. R.; Mártinez-Alonso A.; Táscon, J. M. D.; Int J Coal Geol. 1997, 33, 369. 


\section{CARACTERIZAÇÃO DETALHADA DE MATERIAL DE REFERÊNCIA CERTIFICADO DE CARVÃO BRASILEIRO}

Vera L. V. Fallavena, Cristiane S. de Abreu, Taísi D. Inácio, Marçal Pires e Carla M. N. Azevedo*

Faculdade de Química, Pontifícia Universidade Católica do Rio Grande do Sul, Av. Ipiranga, 6681, 90619-900 Porto Alegre - RS, Brasil

Iolanda D. Fernandes e Lizete S. Ferret

Fundação de Ciência e Tecnologia, Rua Washington Luiz, 675, 90010-460 Porto Alegre - RS, Brasil

M. Rosa Martinez Tarazona

Instituto Nacional do Carvão, C/Francisco Pintado Fe, 26. 33011 Oviedo, Espanha

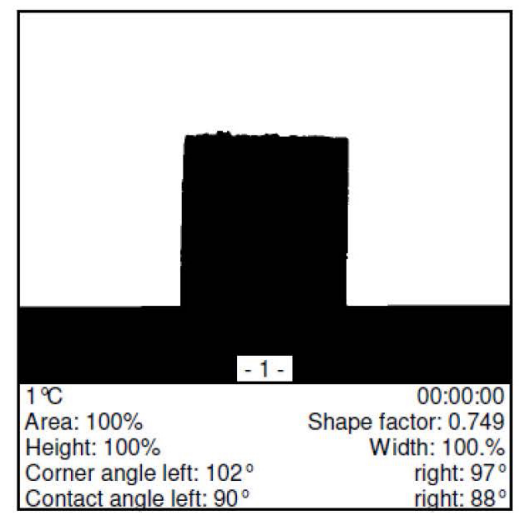

Corpo de prova

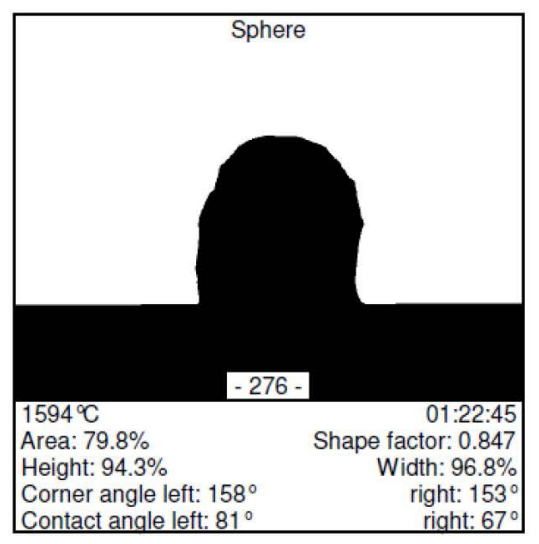

Temperatura de Esfera



Temperatura Inicial de Deformaçãc



Temperatura de Hemiesfera

Figura 1S. Comportamento da cinza de carvão em teste de fusibilidade 

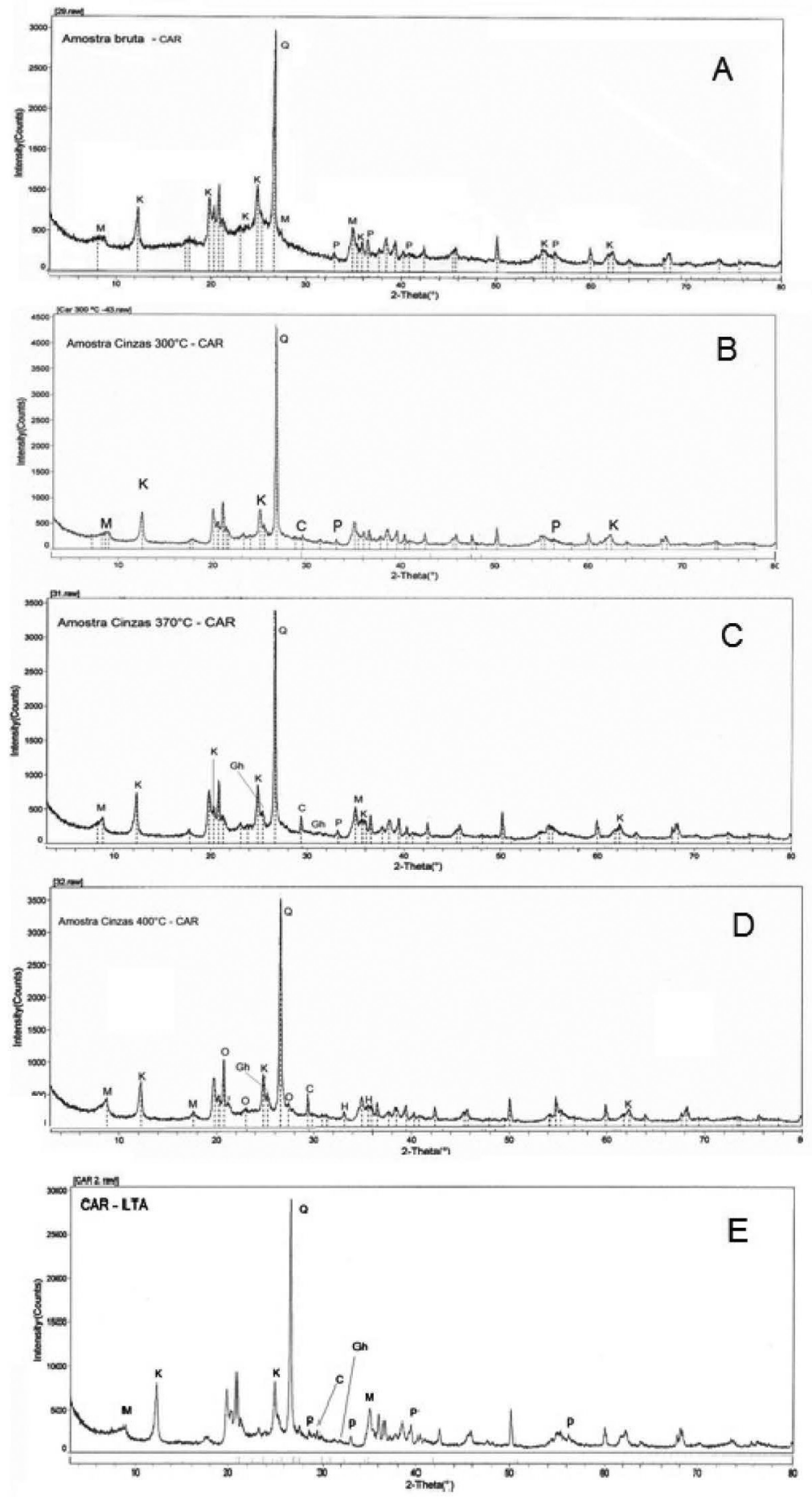

Figura 2S. Difratogramas obtidos para a amostra CAR(MR): (A) amostra bruta, (B) cinzas a $300^{\circ} \mathrm{C},(\mathrm{C})$ cinzas a $370{ }^{\circ} \mathrm{C},(\mathrm{D})$ cinzas a $400{ }^{\circ} \mathrm{C}$ e $(E) \mathrm{LTA}$. $Q-$

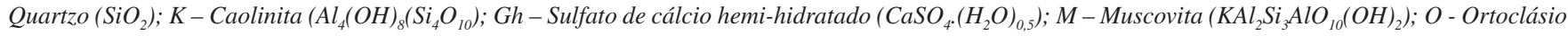
$\left(\mathrm{KAlSi}_{3} \mathrm{O}_{8}\right) ; \mathrm{P}-\mathrm{Pirita}\left(\mathrm{FeS}_{2}\right) ; \mathrm{C}-\mathrm{Calcita}\left(\mathrm{CaCO}_{3}\right) ; \mathrm{H}-\mathrm{Hematita}\left(\mathrm{Fe}_{2} \mathrm{O}_{3}\right)$ 